SOLAR VORTICES AND MAGNETIC FIELDS.' I HEARTILY appreciate the privilege of describing in this lecture-room some of the recent work of the Mount Wilson Solar Observatory. Like so much of the scientific research of the present day, it goes back for its origin to the fundamental investigations of English men of science. The spectroheliograph, which tells us of the existence of solar vortices, is a natural outcome of the application of the spectroscope in astronomy, where Englishmen were foremost among the pioneers. The detection of a magnetic field within these vortices followed directly from Zeeman's beautiful discovery of the influence of magnetism on radiation - a logical extension of the earlier work of Faraday-and from the classic investigations of Crookes and Thomson on the nature of electricity. In reviewing these great advances, investigators in other lands must again and again wonder at the exceptional ability of the English mind to make fundamental discoveries. When these discoveries have been made it. is a comparatively simple matter to utilise them in many departments of science. Americans cannot fail to rejoice that they may share in the traditions of a race which counts among its members the men who have given the Royal Institution its fame.

It is customary to distinguish sharply between the observational and experimental sciences, including astronomy in the former. In physics or chemistry the investigator has the immense advantage of being able to control the conditions under which his observations are made. The astronomer, on the other hand, must be content to observe the phenomena presented to him by the heavenly bodies, and interpret them as best he may. I wish to emphasise the fact, however, that the distinction between these two methods of research is not so fundamental as it may at first sight appear. In I860 a laboratory, in which experiments were conducted for the interpretation of astronomical observations, was established by Sir William Huggins on Upper Tulse Hill. The advantage of imitating celestial phenomena under laboratory conditions was thus appreciated half a century ago. I shall indicate later how important a part such a laboratory plays in the work of the Mount Wilson Solar Observatory. I shall also show that in other ways the astronomer may advantageously follow the physicist, particularly in the choice of observational methods and in the design of instruments of research.

Sun-spots were discovered as soon as Galileo and his contemporaries directed their little telescopes to the sun. In fact, ancient Chinese records indicate that spots of exceptional size had been detected by the naked eye many centuries before. Long after their discovery, the most diverse views were held as to the nature of sun-spots. Sir William Herschel mentioned the uncertainty which had existed prior to his time, remarking that the spots had been variously described as solid bodies revolving about the sun, very near its surface; the smoke of volcanoes; smoke floating on a liquid surface; clouds in the solar atmosphere; the summits of solar mountains, uncovered from time to time by the $e b b$ and flow of a fiery liquid, \&c. In Herschel's own view the spots are to be considered as the opaque body of the sun seen through openings in the luminous atmosphere which envelops it. Indeed. he considered that the sun should be regarded as the primary planet of our system, and even suggested the prohability that it is inhabited. "Whatever fanciful poets might say, in making the Sun the abode of blessed spirits, or angry moralists devise, in pointing it out as a fit place for the punishment of the wicked, it does not appear that they had any other foundation for their assertions than mere opinion and vague surmise; but now I think myself authorised, upon astronomical principles, to propose the Sun as an inhabitable world, and am persuaded that the foregoing observations, with the conclusions I have drawn from them, are fully sufficient to answer every obiection that mav be made against it." 2

Sir John Herschel did not abandon the idea of an opaque solar globe, but suggested that hurricanes or tornadoes

1 Discourse delivered at the Royal Institution on Friday, May 14, by rof. George Fi,

${ }^{2}$ William Herschel, "On the Nature and Construction of the Sun and Fixed Stars," p. 20.

NO. 2088, VOL. 82] might account for the piercing of the two strata of luminous matter which ordinarily conceal this globe. "Such processes cannot be unaccompanied by vorticose motions, which, left to themselves, die away by degrees and dissipate-with this peculiarity, that their lower portions come to rest more speedily than their upper, by reason of the greater resistance below, as well as the remoteness from the point of action, which lies in a higher region, so that their centre (as seen in our water-spouts, which are nothing but small tornadoes) appears to retreat upwards. Now, this agrees perfectly with that which is observed during the obliteration of the solar spots, which appear as if filled in by the collapse of their sides, the penumbra closing in upon the spot, and disappearing after it."

We now know that sun-spots are brighter than the brightest arc light, and that their apparent darkness is merely the result of the contrast with the intensely brilliant surface of the photosphere. We also know that the sun is a gaseous globe, attaining a temperature of about $6000^{\circ}$ at its surface, and perhaps millions of degrees at its centre. If we examine a large-scale photograph of a sun-spot we see that it consists of a dark central region, called the umbra, and a surrounding area, decidedly less dark, called the penumbra. The structure of a spot, as this admirable photograph by Janssen shows, is granular, like that of the photosphere. In the penumbra these granulations seem to group themselves more or less radially, as though under the influence of some force directed toward or away from the umbra. Unfortunately, direct photographs of the sun have not yet attained such perfection as to show the most minute details of sun-spots. To appreciate these, we must have recourse to the exquisite dratvings of Langley, the truthful quality of which is recognised by every astronomer who has observed sun-spots under favourable conditions. We shall see that the characteristic structure represented by these drawings is repeated, on a far greater scale, in the higher regions of the solar atmosphere disclosed on recent spectroheliograph plates.

Since the time of Sir John Herschel, many astronomers have pronosed vortex theories of sun-spots. One of the first of these is the theory of Faye, who supposed the whirling motion to be the direct result of the peculiar law of the sun's rotation. This law was discovered by Carrington, who found from observations of spots near the equator that the sun completes a rotation in about twentyfive days, while the motion of spots at a latitude of $40^{\circ}$ indicated the time of rotation to be nearly two days longer. Thus, as the rotation period increases toward the poles, the photosphere at the northern and southern boundaries of a sun-spot must move at different velocities (assuming the law of the sun's rotation to be the same as that of the spots). This difference in velocity would tend to set up whirling motions, clockwise in the southern hemisphere and counter-clockwise in the northern hemisphere. Sun-spots, in Faye's opinion, are the visible evidences of such whirls.

This theory has had many supporters, but it is now generally agreed that the difference in the rotational velocity of adjoining regions of the photosphere is not nearly sufficient to account for the observed phenomena. Secchi, one of the most assiduous observers of solar phenomena, was strongly opposed to Faye's theory. $\mathrm{He}$ pointed out that about 6 per cent. of the spots he observed gave some cvidence of cyclonic action, but in the vast majority of cases such forms as Faye's theory seemed to demand were lacking. We nevertheless owe to Secchi a most striking drawing of a sun-spot vortex.

When the spectroheliograph was first systematically applied to solar research in 1802 , many rival theories of sun-spots occupied the field. Since the function of this instrument is to photograph the phenomena of the invisible solar atmosphere, it might be hoped that the results would throw much light on the nature of sun-spots. For many years, however, this hope was not realised. The first monochromatic images of the sun were made with the $K$ line of calcium. If we compare such an image with a direct photograph of the sun, made in the ordinary wav, we see that the sun-spots are surrounded and frequentiy covered by vast clouds of luminous calcium vapour. These attain elevations of several thousand miles above the sun's surface, but they must not be confused with the promin. ences, which ascend to much higher elevations. When 
observed at the sun's limb, the bright calcium flocculi, as these luminous clouds are called, are so low, in comparison with the prominences, that they can hardly be detected as elevations. Thus our knowledge of the calcium flocculi must be derived mainly from the study of spectroheliograph plates, which show them in projection on the disc. I must not omit to mention, however, that the calcium vapour rises to the highest parts of the prominences, and that this higher and cooler vapour frequently indicates its presence on spectroheliograph plates in the phenomena of dark flocculi. These are relatively inconspicuous, however, and need not be discussed here. ${ }^{1}$

It soon appeared that the average photograph of bright calcium flocculi could not be counted upon to indicate the existence of definite streams or currents in the solar atmosphere. In 1903 the hydrogen flocculi were photographed for the first time. By comparing these flocculi with the corresponding calcium flocculi we see that, in general, dark regions on the hydrogen image agree approximately in form with bright regions on the calcium image. This might appear to indicate that hydrogen is absent in the regions where calcium is most abundant. An investigation of the question, however, does not lead to this conclusion. Dark hydrogen flocculi seem to mark those regions on the sun's disc where hydrogen is present as an absorbing medium, which reduces the intensity of the light coming through it from below. In certain areas, where the temperature is higher or the condition of radiation otherwise different, the hydrogen flocculi are bright. In many cases eruptions are in progress at these points, but in others the difference in brightness is apparently not the direct result of eruptive action.

The hydrogen flocculi, thus photographed with the lines $\mathrm{H} \beta, \mathrm{H} \gamma$, or $\mathrm{H} \delta$, differ in many respects from the calcium flocculi. Not only do they usually appear dark, where the calcium flocculi are bright: their forms exhibit striking peculiarities, which are absent or much less conspicuous in the case of calcium. The appearance of the calcium fiocculi resembles that of floating cumulus clouds in our own atmosphere; their capricious changes in form reveal the operation of no simple law. But the hydrogen flocculi, on the contrary, exhibit a definiteness of structure in striking contrast to this appearance. Some of the photographs strongly remind us of the distribution of iron filings in a magnetic field, and suggest that some unknown force is in operation.

Such was the condition of the subject when the red $\mathrm{Ha}$ line of hydrogen was first applied to the photography of the flocculi, on Mount Wilson, in March, 1908. The calcium and hydrogen flocculi had been studied for several years, and much had been learned as to their nature and their motions. It had been found, for example, that the calcium flocculi observe the same law of rotation that governs the motions of sun-spots, while the hydrogen flocculi apparently follow a different law, in which the decrease in the angular rotational velocity from the equator toward the poles is much less marked. The latter result is in harmony with the investigations of Adams, whose accurate measures of the approach and recession of the hydrogen at the eastern and western limbs of the sun offer but little evidence of equatorial acceleration on the part of this gas. For this and other reasons it had been concluded that the hydrogen shown in such photographs reaches a higher level than the vapours of the bright $(\mathrm{H})$ calcium flocculi. The region of the atmosphere previously explored with the spectroheliograph was nevertheless confined (except in the case of eruptions and dark calcium flocculi) to a comparatively low level, lying within a few thousand miles of the photosphere. What might be expected if a still higher region could be satisfactorily photographed in proiection on the disc?

The red line of hydrogen offered the means of disclosing the phenomena of this higher atmosphere. As it may not immediately appear why different lines, caused by the radiation of the same gas, should not give precisely similar photographs, a brief reference to the aspect of a prominence in the red and blue hydrogen lines may be advantageous. Here are two photographs of the same prominence, seen in elevation at the sun's limb, one made

1 Eruptive prominences are also recorded on the disc as bright floccul.

NO. 2088, VOL. 82] with $H \alpha$, the other with $H \delta$. As the red line is very bright, even in the highest regions, the photograph taken with its aid shows the entire prominence. $\mathrm{H} \delta$, on the other hand, is relatively weak at the higher leveks, and consequently only the lower and brighter parts of the prominence are weil recorded when this line is used. If, now, we suppose ourselves immediately above such a prominence, at a point where we observe it in projection against the disc, it is evident that the character of the hydrogen lines must depend upon their brightness at different levels. As we know that, speaking generally, absorption is proportional to radiation, the amount of light absorbed in the upper part of the prominence will be much greater for $\mathrm{H} \alpha$ than for $\mathrm{H} \delta$. Hence the average level represented by the absorption of $\mathrm{H} \alpha$ will be higher than the average level represented by $H \delta$, since the higher gases play a more important part in the production of the former line. We may therefore expect that photographs of the sun's disc, taken with the light of $\mathrm{Ha}$, will show the dark areas corresponding to absorption in the prominences much more clearly than photographs taken with $\mathrm{H} \delta$. Moreover, since $\mathrm{H} \alpha$ is stronger than $\mathrm{H} \delta$ in the upper chromosphere, in regions where no prominences are present, the average level represented by this line will, in general, be higher than that represented by $\mathrm{H} \delta$. A comparison of two photouraphs of the sun's disc, made with the lines in question, will suffice to make this clear. This

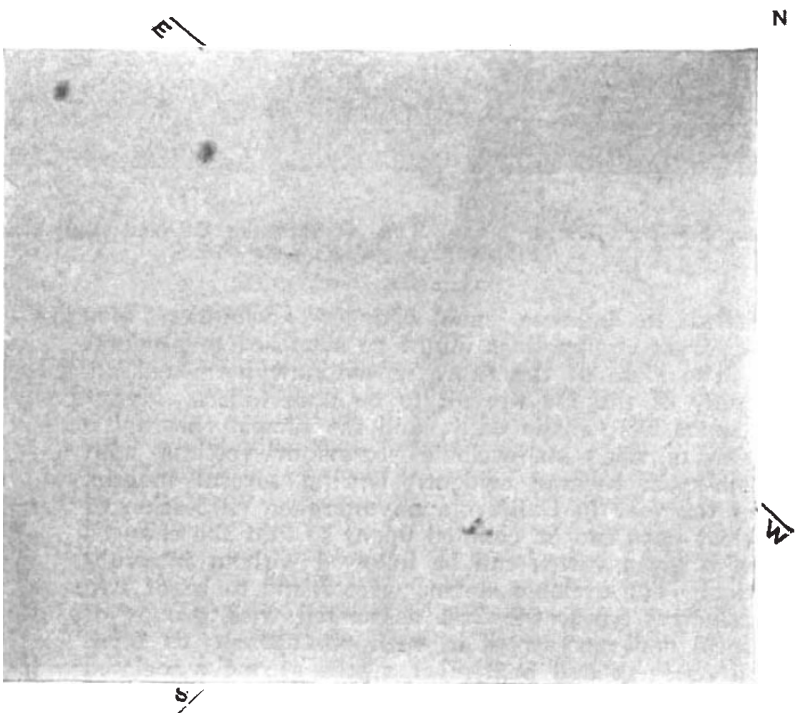
Fig. I. -Direct Photograph of Sun-spot Group. x908, April 30, 6h. $25 \mathrm{~m}$

erotmous group of prominences, stretching for several hundred thousand miles across the sun, is much more clearly indicated by $\mathrm{H} \alpha$ than by $\mathrm{H} \delta$. In general, the hydrogen flocculi are stronger and more distinct when phiotographed with $\mathrm{Ho}$, and there are some regions which appear bright with $\mathrm{H} \alpha$ and dark with $\mathrm{H} \delta$. This latter peculiarity probably has an important bearing upon the similar behaviour of hydrogen in certain stars and nebulæ, but a discussion of this question cannot be undertaken here.

The first of the $\mathrm{H} \alpha$ photographs gave strong hopes of a substantial advance in our knowledge of the solar atmosphere. The sharpness and comparatively strong contrast of these flocculi, and the evidences of definite structure and clearly defined stream lines which they revealed, were highly encouraging. The work was begun during the disturbed weather of the rainy season, when the definition of the solar image is never of the best. On April 30, 1908, the first photographs were secured under the fine atmospheric conditions which prevail in the dry season. $\Lambda$ direct photograph (Fig. 1) shows a small and insignificant gioup of sun-spots, which would not seem, without other indications, to merit special attention. The next phulograph (Fig. 2) shows that an enormous calcium flocrulus 
occupied this resion of the sun, but its form was in no nise remarkable, and afforded no evidence of the phenomena brought to light by the $\mathrm{H} \alpha$ photograph. The structure recorded with the aid of the latter line (Fig. 3) recalls Langley's sun-spot drawings, and suggests the operation of some great force related to the sun-spot group. The same cyclonic structure had been less satisfactorily recorded on the previous day, but a comparison of the two photographs

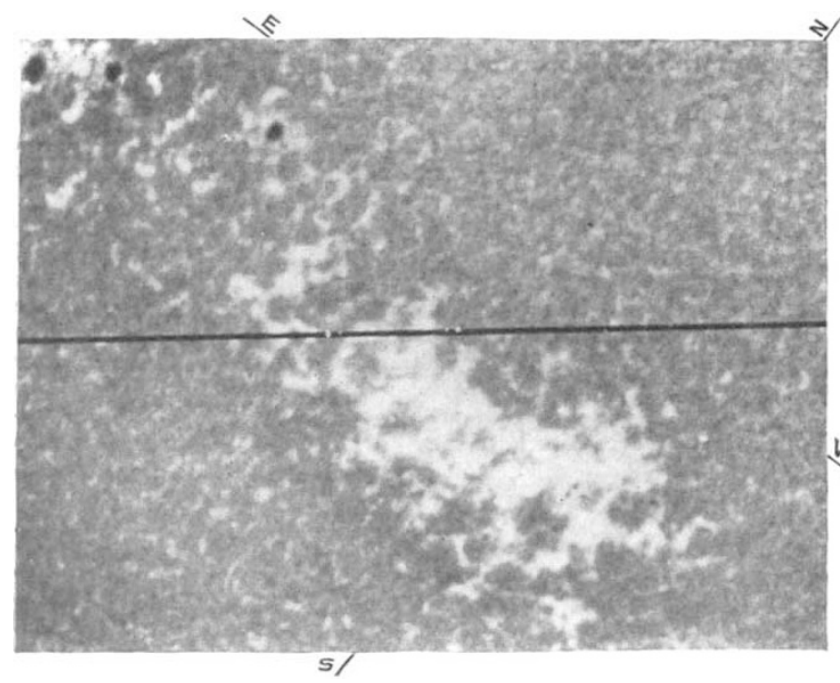

Fig. 2.-Same Region of the Sun, s'owing the Calcium $\left(\mathrm{H}_{2}\right)$ Flocculi, rgos, April 3o, 4 h. 43 m. p.m. P.S.T.

failed to indicate such changes as motion along the apparent stream lines might be supposed to produce.

The close of the rainy season now permitted an active study of the $\mathrm{H} \alpha$ flocculi to be undertaken. Many photogtaphs were made daily, and the almost constant association of apparent cyclonic storms or vortices with sun-spots became evident. During several months of the year in California an unbroken succession of clear days can be counted upon, so that the changes of a given vortex can be followed without interruption. The cyclonic storms were found to be of two principal types, the first associated with groups of spots and represented in such photographs as those of April 30 and September 2, the second associated with single spots, and resembling a simple vortex, as illustrated in the photographs of September 9 and October 7, 1908 (Fig. 4). The appearance of these simple vortices is such as to indicate rotation in a clockwise direction in the southern hemisphere, and in a counter-clockwise direction in the northern hemisphere (assuming the direction of motion to be inward toward the spot). However, this cannot be taken as a general law, corresponding to the law of terrestrial cyclones. Indeed, many instances have been found of closely adjoining spots, in the same hemisphere and frequently in the same spot-group, having magnetic fields of opposite polarity, produced by vortices rotating in opposite directions.

In some cases, at least, these vortices seem to exercise a powerful attraction on the surrounding gases, as a series of photographs taken on June 3 , 1908, illustrates. A long dark hydrogen prominence, first photographed in elevation at the sun's limb on May 28 , had advanced half-way across the solar disc. It lay at the outer boundary of a well-defined vortex, céntred on a sun-spot. This spot had been gradually separating into two parts, and on June 3 the separation was complete. The first photograph of a series of nine was made on this day at $4 \mathrm{~h} .58 \mathrm{~m}$. Several successive photographs indicated no appreciable change, but one taken at $5 \mathrm{~h}$. $07 \mathrm{~m}$. showed that the prominence was developing an extension toward the spot. At $5 \mathrm{~h}$. $14 \mathrm{~m}$. this had assumed the appearance illustrated in the next photograph, and eight minutes later, when the last photoNO. 2088, VOL. 82] graph of this series was taken, the extension had almost reached the spot. It will be seen that it divided into two parts, which indicates that each umbra was a centre of attraction. The average velocity of the motion toward the spot was more than $100 \mathrm{~km}$. per second. Later photographs, made on the following days, show a ring of bright hydrogen surrounding the spots, suggesting that the comparatively cool hydrogen carried down into the spots was re-heated and returned to the surface, after escaping from the lower end of the vortex. We thus seem to be observing some of the phenomena of an actual vortex in the sun; but it must not be supposed that cases of this kind are common. In many instances the hydrogen flocculi do not appear to move rapidly toward or away from spots, but undergo changes of intensity, as though the physical condition of the gas were constantly changing; but before proceeding further with a discussion of these sun-spot vortices, let us turn to another phase of the subject, which will afford much new information indispensable for this purpose.

We are all familiar with the effect produced by passing an electric current through a wire helix. The lines of force of the resulting magnetic field are parallel to the axis of the helix, and its intensity is determined by the diameter of the helix, the number of turns of wire, and the strength of the current. We also know, from Rowland's experiment, that the rapid revolution of an electrically charged body will produce a magnetic field. Thus, if a sufficient number of electrically charged particles were set into rapid revolution by the solar vortices, a magnetic field should result. What warrant have we for assuming the existence of charged particles in the sun, and how could such a field be detected?

Let me pass rapidly in review a series of phenomena with which you are all familiar. Sir William Crookes showed in this lecture-room, so long ago as I 879 , that the negative pole of a vacuum tube sends out a stream of particles, capable of setting a light windmill in rotation, and deviated from their straight path when under the influence of a magnetic field. He has kindly consentril to show the same tube again to-night: you nor see the

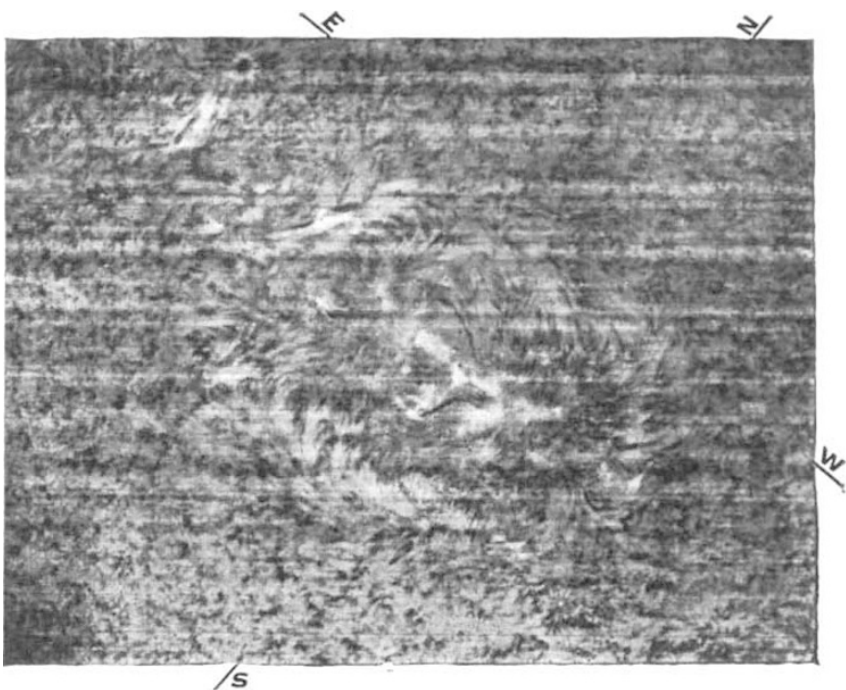

FIG. -- - Same Region of the cul, showing the Hydrogen (Ha) Flocculi. Igo8, April 3), 5h, o6m. p.m. P.S.l.

effect upon the screen. The recent work of Sir Joseph Themson and others has proved that these are negatively charged particles, called " corpuscles" or "electrons," and that their mass is about $1 / 1700$ of the mass of an atom of hydrogen. Moreover, Thomson has shown that at low pressures these corpuscles are given off from a hot wire or from the carbon filament of an incandescent lamp. He has also denonstrated that this property of emitting 
corpuscles at high temperature is common to carbon and to metals, whether in the solid or in the vaporous condition. Thus we have warrant for the belief that the sun, composed of just such elements as constitute the earth, must emit great numbers of these corpuscles. As Thomson has estimated that the rate of emission of a carbon filament at its highest point of incandescence may amount to a current equal to several amperes per square centimetre of surface, we can hardly be mistaken in assuming the existence of still more powerful currents in the sun. The emission of negatively charged particles implies the emission of positively charged particles, but in laboratory experiments, because of unequal rates of diffusion or other causes, charges of one sign are always found to be in excess. We thus have reason to believe that powerful magnetic fields may result from the revolution of these particles in the solar vortices.

In seeking a means of detecting such fields, let us first recall Faraday's discovery of the effect of magnetism on light, made at the Royal Institution in 1846 . This discovery relates to the rotation of the plane of polarisation of light when passed through a plate of dense glass in a strong magnetic field. Although Faraday, in what was said to be his last experiment, endeavoured to detect the polarised in planes at right angles to one another. A Nicol prism, standing at a certain angle, will transmit one of these plane polarised beams. and cut off the other. Turning the Nicol through $90^{\circ}$ will cause the component previously cut off to be transmitted, and the other to be stopped.

Consider a sun-spot at the centre of the solar disc, and suppose it to be produced by a vortex, the axis of which lies on the line passing from the eye of the observer through the spot to the centre of the sun. In these circumstances, if a strong magnetic field is produced by the vortex, the spectral lines due to vapours lying within this field should be widened or transformed into doublets. Moreover, the light of the components of these doublets should be circularly polarised in opposite directions. This would be true if the spot vapours were emitting bright lines, identical in character with those emitted by a radiating vapour between the poles of a magnet. The experiments of Zeeman, Cotton, König, and others, show, however, that dark lines, produced by the absorption of the spot vapours, should behave precisely in the same way as bright lines.

The spectrum of a sun-spot was observed for the first time by Locikyer in 1866 . He found that many of the lines of the solar spectrum were widened where they crossed the spot, and the observation of these widened lines has been carried on systematically by many observers ever since. Conspicuous among these observers was Young, whose last observations were made with a powerful grating spectroscope attached to the 23 -inch Princeton refractor. This instrument showed that some of the spot lines are close doublets. Dr. Walter M. Mitchell, who at first worked in conjunction with Prof. Young, and later by himself, gave special attention to these double lines, which he found to be particularly numerous at the red end of the spectrum. He called them "reversals," and the existing evidence favoured the view that they were produced by the radiation of a hotter layer of vapours overlying the spot, which would give rise to a narrow bright line at the centre of the widened dark line. True reversals of this kind actually seem to occur in the case of $\mathrm{H}$ and $\mathrm{K}$ and other lines in the spot spectrum, and it was therefore natural that Mitchell should attribute the similar phenomena of the spot doublets to a similar cause. It was generally supposed that the widening of the dark lines was due to the increased density of the spot vapours. The diverse character of the lines in the sun-spot spectrum is well illustrated by this drawing, which is due to Mitchell. In addition to the ordinary

to Mitchell. In addition to the ordinary
widened and "reversed "lines we find cases where a dark
central line is accompanied by wings, others in which lines effect of magnetism on the lines of the spectrum, he failed because the apparatus then available was not sufficiently powerful. In 1896 Prof. Zeeman examined with a large spectroscope the two yellow lines emitted by sodiun vapour in a flame between the poles of a powerful magnet. Observing in the direction of the lines of force, he saw that the sodium lines widened when the magnet was excited. Subsequently, with more powerful apparatus, he found that a single line, when observed under the above conditions, is split into two components by a magnetic field. The distance between the two components is a measure of the strength of the field; but the most characteristic quality of these double lines, which distinguishes them from double lines produced by any other known means, is the fact that the light of the two components is circularly polarised in opposite directions. If, then, we encounter a double line in the spectrum of any substance, and suspect it to be due to a magnetic field, we must apply the test for circular polarisation.

The simplest means of testing for circularly polarised light is to transform it into plane polarised light by passing it through a quarter-wave plate or a Fresnel rhomb. In the case of a Zeeman doublet, we would then have issuing from the rhomb the light of the two components, NO. 2088, VOL. 82] are thinned or completely obliterated, \&c.

\section{RECENT AGRICULTURAL PUBLICATIONS FROM THE WEST INDIES.}

THE imperial Department of Agriculture for the West Indies issue three periodical publications:-(I) the West Indian Bulletin, a quarterly scientific journal constaff, or papers read at agricultural conferences; (2) the Agricultural Neres, a fortnightly paper, published at one on subjects of importance to planters; (3) a series of bulletins, each containing detailed information on some special subject. In addition, reports are issued on the work done at the different experiment stations on some of the islands, and the Department of Agriculture for Jamaica issues a separate bulletin of its own.

During the present year the last number of vol. ix. of the West Indian Bulletin and the first of vol. $\mathrm{x}$. have been published. The timbers of Jamaica are described at
(To be continued.) taining papers or reports by members of the scientific penny only, containing short articles in popular language 